clustering with ability to identify upregulation of cancer specific genes in malignant relative to benign nodes (notably EGFR, HGFR/ c-met and HER2 were among genes most upregulated).

Conclusion We demonstrate the feasibility of RNA extraction and GEP on EBUS-derived lymph node cytological aspirates and show differences in gene expression profiles between benign and tumourinfiltrated lymph node mRNA. Further studies on larger patient cohorts are necessary to identify expression profiles that can robustly differentiate benign from malignant lymph nodes in NSCLC.

\section{S58 BETA-CATENIN DETERMINES TRACHEAL CELL FATE AND SQUAMOUS LUNG CANCER PROGRESSION BY MODULATING INTERCELLULAR ADHESIVENESS}

doi:10.1136/thoraxjnl-2011-201054b.58

\begin{abstract}
${ }^{1} \mathrm{~A}$ Giangreco, ${ }^{1} \mathrm{~L}$ Lu, ${ }^{1} \mathrm{C}$ Vickers, 'E llieva, ${ }^{1} \mathrm{~K}$ Groot, ${ }^{1} \mathrm{~J}$ George, ${ }^{1} \mathrm{~A}$ Nicholson, ${ }^{1} \mathrm{E}$ Sage, ${ }^{2} \mathrm{~F}$ Watt, ${ }^{1} \mathrm{~S}$ Janes. ${ }^{1}$ University College London, London, UK; ${ }^{2}$ Cancer Research UK, Cambridge, UK
\end{abstract}

Human lung cancers including squamous cell carcinoma (SCC) are a leading cause of death, and while evidence suggests that basal stem cells drive SCC initiation and progression, the mechanisms regulating these processes remain unknown. In this study we show that $\beta$ catenin signalling regulates basal stem cell fate and subsequent SCC progression. In a cohort of preinvasive SCCs we established that elevated basal stem cell $\beta$-catenin signalling is positively associated with increased disease severity, epithelial proliferation, and reduced intercellular adhesiveness. We demonstrate that transgene-mediated $\beta$-catenin inhibition within keratin 14-expressing basal stem cells delayed normal airway repair while basal cell-specific $\beta$-catenin activation increased cell proliferation, directed differentiation, and promoted an epithelial-to-mesenchymal transition (EMT) that included increased Snail transcription and reduced E-cadherin-mediated adhesiveness. These effects were recapitulated in normal human bronchial epithelial cells in vitro following both pharmacological $\beta$ catenin activation and E-cadherin inhibition, and mirrored our findings in preinvasive SCCs. Overall this data shows that airway stem cell $\beta$-catenin modulates cell adhesiveness to determine cell fate and its mis-expression is a key step in the development of human lung cancer.

\section{S59 CHEMO-IMMUNOTHERAPY OF MESOTHELIOMA: DEPLETION OF ESTABLISHED, SUPPRESSIVE CD4 T CELLS IS CRITICAL TO ACHIEVING CURES IN A MURINE MODEL}

doi:10.1136/thoraxjnl-2011-201054b.59

H J Steer, A Cleaver, A Nowak, B Robinson, R Lake. University of Western Australia, Perth, Australia

Introduction Mesothelioma is incurable and new treatment strategies are urgently needed. It has long been recognised that malignant cells can be attacked by the immune system but immunotherapy is only starting to emerge as an additional modality of treatment for cancer. The crucial role that helper CD4 T cells play in orchestrating CD8 $\mathrm{T}$ cell and other anti-tumour responses has only recently been appreciated. However the CD4 population contains effector and suppressive subsets with diverse and opposing functions. Although the effects of pemetrexed on tumour immunity are still being defined, gemcitabine is known to synergise with immunotherapy by causing tumour cell death, releasing tumour antigen and depleting suppressive immune cells. We investigated the role that different types of CD4 cell play in CD8 anti-tumour immune responses and how the CD4 response could be best manipulated to optimise outcomes to chemo-immunotherapy.

Methods Thy1.2+ve balb/c mice bearing mesothelioma tumours expressing the neoantigen haemagglutinin (HA), were treated with gemcitabine and adoptive transfer of tumour antigen (HA) specific thy1.1+ve CD8 T cells and thy1.1+ve CD4 cells which had been differentiated in vitro into Th1s, Th2s, Th17s or regulatory $\mathrm{T}$ cells (Tregs). The activity of the adoptively transferred cells in vivo was tracked by identifying recovered thy1.1+ve cells by flow cytometry. Results In the prophylactic treatment setting, in vitro differentiated tumour antigen specific Th1s led to rejection of tumours inoculated 1 day later in around $40 \%$ of mice. However there was minimal activity against established, gemcitabine-treated tumours. Recovery of adoptively transferred CD8 $\mathrm{T}$ cells revealed that in established tumours, CD4s were unable to help cognate CD8 cells expand and infiltrate tumours. However if endogenous CD4s were depleted prior to treatment then CD8 tumour infiltration was significantly increased and cures were seen in around two-thirds of mice.

Conclusions These results suggest that once a tumour is established, an over-riding endogenous suppressive CD4 response prevents effector CD4s from helping CD8 $\mathrm{T}$ cells to eradicate tumours Removal of suppressive CD4 cells was critical to achieving cures with chemo-immunotherapy.

\section{S60 MESENCHYMAL STEM CELLS EXPRESSING TRAIL INDUCE APOPTOSIS IN MALIGNANT PLEURAL MESOTHELIOMA}

doi:10.1136/thoraxjnl-2011-201054b.60

E Sage, K Kolluri, A Giangreco, S Janes. University College London, London, England

Background Malignant pleural mesothelioma (MPM) is an aggressive fatal cancer caused by asbestos exposure. Current treatments are ineffective with an average survival of 4-18 months. Mesenchymal stem cells (MSCs) migrate to tumours and incorporate into tumour stroma making them good vehicles for the delivery of anticancer therapies. TNF-related apoptosis inducing ligand (TRAIL) is a transmembrane protein that selectively induces apoptosis in malignant cells without affecting healthy tissues. In this study I tested the hypothesis that MSCs modified to express TRAIL (MSCTRAIL) would cause MPM cell death.

Methods Human MSCs were transduced with a lentiviral vector containing TRAIL IRES-GFP under the control of a tetracycline dependent promoter. Successful transduction was measured using flow cytometry and immunoblotting. The biological activity of MSCTRAIL was determined using co-culture experiments. $5 \times 10^{5}$ MPM cells were stained withDiI and plated with $5 \times 10^{5}$ MSCTRAIL cells. After $24 \mathrm{~h}$ doxycycline $(10 \mu \mathrm{g} / \mathrm{ml})$ was added to induce TRAIL production and left for $48 \mathrm{~h}$. Both cells and supernatant were collected and stained with Annexin V and DAPI to detect apoptosis and death respectively onflow cytometry.

Results MSCs were successfully transduced with TRAIL with $96 \%$ showing GFP positivity on flow cytometry. Seven human MPM cell lines were tested with $6 / 7$ (86\%) being sensitive to MSCTRAIL. JU77 was highly sensitive with an increase in apoptosis from $10.32 \pm 2.34$ to $48.73 \pm 4.3$ (percentage \pm SEM, $p \leq 0.0001$ ), while MSTO-211H and ONE 58 showed increases from $3.46 \pm 0.81$ to $27.68 \pm 1.1$ and $8.92 \pm 0.05$ to $32.93 \pm 1.08$ respectively ( $p \leq 0.001$ )

Conclusions MSCs can be successfully transduced to produce TRAIL and can induce significant levels of apoptosis in the majority of MPM cell lines tested.

\section{Pleural infection and pneumonia}

\section{S61 RAPID SCORE: THE DEVELOPMENT OF A VALIDATED CLINICAL SCORE IN PLEURAL INFECTION, TO IDENTIFY AT PRESENTATION THOSE AT RISK OF POOR OUTCOME}

doi:10.1136/thoraxjnl-2011-201054b.61

${ }^{1} \mathrm{~N}$ M Rahman, ${ }^{2} \mathrm{~B}$ C Kahan, ${ }^{3} \mathrm{R}$ Miller, ${ }^{4} \mathrm{~N}$ A Maskell. ${ }^{1}$ Oxford Respiratory Trials Unit, Oxford Centre for Respiratory Medicine, Oxford, UK; ${ }^{2}$ MRC Clinical Trials Unit, London, 